\title{
Nanotube formation from a sodium titanate powder via
}

\section{low-temperature acid treatment}

\author{
Chien-Cheng Tsai and Hsisheng Teng*
}

Department of Chemical Engineering and Center for Micro/Nano Science and

Technology, National Cheng Kung University, Tainan 70101, Taiwan

\section{Supporting Information}

Figure S1 The structure models of titanates: a) the monoclinic system with $\alpha=\gamma=$ $90^{\circ}, \beta \neq 90^{\circ}$; b) the orthorhombic system with $\alpha=\beta=\gamma=90^{\circ}$.

Figure S2 $\mathrm{N}_{2}$ adsorption (full symbol)-desorption (empty symbol) isotherms for the titanate specimen obtained from the solid-state synthesis (Sodium-Titanate) and those obtained after the consecutive post-treatments: the hydrothermal treatment in $\mathrm{NaOH}$ (Sodium-Titanate-H), the acid treatment to $\mathrm{pH}=6$ (Sodium-Titanate-HA), and the acid treatment to $\mathrm{pH}=1.7(\mathrm{NT})$. 

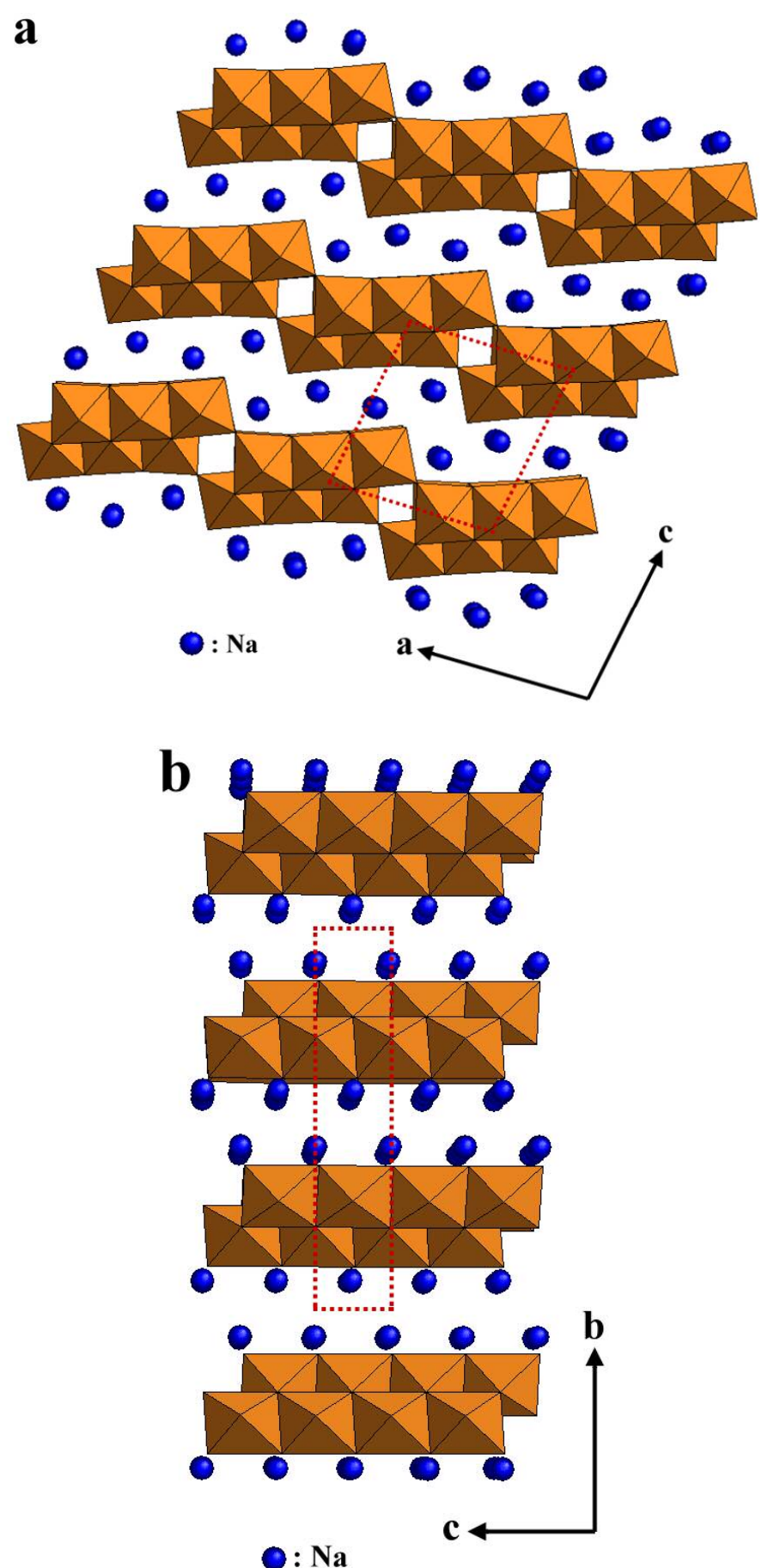

Figure S1 The structure models of titanates: a) the monoclinic system with $\alpha=\gamma=$ $90^{\circ}, \beta \neq 90^{\circ}$; b) the orthorhombic system with $\alpha=\beta=\gamma=90^{\circ}$. 


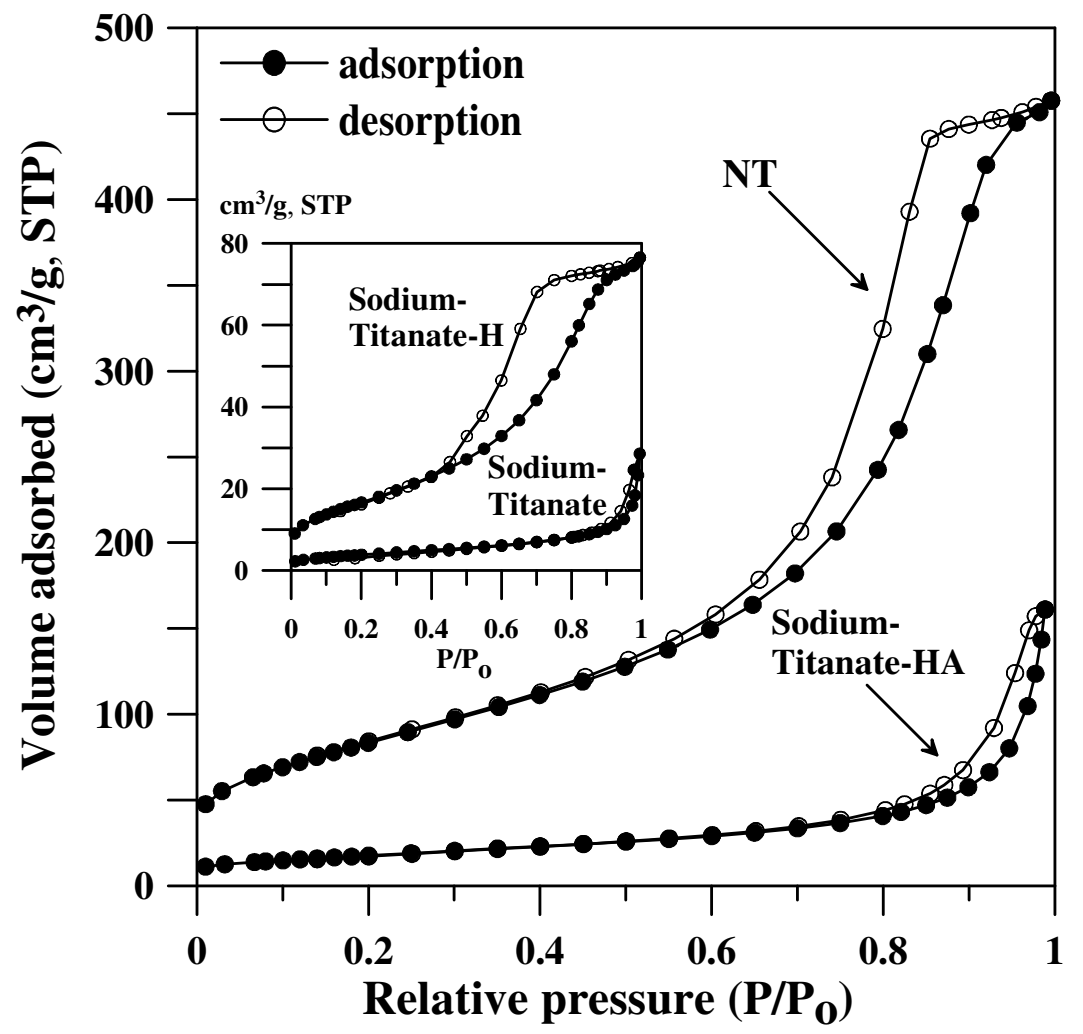

Figure $\mathbf{S} 2 \mathrm{~N}_{2}$ adsorption (full symbol)-desorption (empty symbol) isotherms for the titanate specimen obtained from the solid-state synthesis (Sodium-Titanate) and those obtained after the consecutive post-treatments: the hydrothermal treatment in $\mathrm{NaOH}$ (Sodium-Titanate-H), the acid treatment to $\mathrm{pH}=6$ (Sodium-Titanate-HA), and the acid treatment to $\mathrm{pH}=1.7(\mathrm{NT})$. 